\title{
Medicalización del TDAH en Argentina. Reflexiones sobre tendencias globales y especificidades locales a través del estudio del metilfenidato y la atomoxetina
}

\author{
Eugenia Bianchi \\ Silvia A. Faraone $e^{2}$ \\ Flavia C. Torricelli
}

\section{Resumen}

El objetivo general es contribuir a una mirada panorámica del fenómeno de la medicalización del TDAH en Argentina, y a las discusiones acerca de la diseminación de saberes sobre la infancia.

I Dra. En Ciencias Sociales, Universidad de Buenos Aires, Mgs. en Investigación en Ciencias Sociales, Universidad de Buenos Aires, Lic. en Sociología, Universidad de Buenos Aires. Investigadora Adjunta en Consejo Nacional de Investigaciones Científicas y Técnicas (CONICET, Argentina). Profesora asistente en Universidad de Buenos Aires, Facultad de Ciencias Sociales. Investigadora en Instituto de Investigaciones Gino Germani, Facultad de Ciencias Sociales, Universidad de Buenos Aires. Co-coordinadora del Grupo de Estudios Sobre Salud Mental y Derechos Humanos (GESMyDH) e Investigadora miembro del Área de Salud y Población. E-mail: eugenia.bianchi@gmail.com

2 Dra. en Ciencias Sociales, Universidad de Buenos Aires. Mgs. en Salud Pública, Universidad de Buenos Aires. Lic. en Trabajo Social, Universidad de Buenos Aires. Profesora adjunta en Universidad de Buenos Aires, Facultad de Ciencias Sociales. Investigadora en Instituto de Investigaciones Gino Germani, Facultad de Ciencias Sociales, Universidad de Buenos Aires. Coordinadora del Grupo de Estudios Sobre Salud Mental y Derechos Humanos (GESMyDH) e Investigadora miembro del Área de Salud y Población. E-mail: silfaraone@gmail.com

3 Dra. en Psicología, Universidad de Buenos Aires, Lic. en Psicología. Universidad de Buenos Aires. Profesora asistente en Universidad de Buenos Aires, Facultad de Ciencias Sociales, Investigadora en Instituto de Investigaciones Gino Germani, Facultad de Ciencias Sociales, Universidad de Buenos Aires. Integrante del Grupo de Estudios Sobre Salud Mental y Derechos Humanos (GESMyDH). E-mail: flvtorri@gmail.com

\section{(cc) Br}

Direito autoral e licença de uso: Este artigo está licenciado sob uma Licença Creative Commons. Com essa licença você pode compartilhar, adaptar, para qualquer fim, desde que atribua a autoria da obra, forneça um link para a licença, e indicar se foram feitas alterações. 
El análisis se enmarca en estudios sobre la transformación y expansión de la medicalización en el siglo XXI, y se abordan los procesos de diagnóstico y tratamiento del TDAH. El artículo presenta datos de I) producción y consumo mundial de metilfenidato, y datos de Argentina de 2) importación de metilfenidato como monodroga (2003-2016); 3) envases dispensados de atomoxetina y metilfenidato (2005-2010 y 2012-2016). Estos datos se triangulan con fuentes cualitativas: 84 entrevistas semiestructuradas, individuales y grupales, entre 2007 y 2017, a profesionales de la salud con actividad clínica, docente y de investigación de Ciudad de Buenos Aires, Tierra del Fuego, Corrientes y Salta. Se describen y analizan tendencias argentinas c onsonantes y disonantes con estos procesos a nivel mundial.

Palabras clave: Medicalización. Déficit de atención e hiperactividad. Metilfenidato. Atomoxetina. Argentina.

\section{Introducción}

El trastorno por déficit de atención e hiperactividad (TDAH) y las problemáticas a él vinculadas lo posicionan como uno de los diagnósticos más persistentemente estudiados por las perspectivas sociales críticas. En particular, los trabajos de Peter Conrad y sus colaboradores llevan casi cinco décadas de estudio del TDAH como ejemplo prototípico de las transformaciones en los procesos de medicalización de la sociedad (CONRAD, 1975; CONRAD; SCHNEIDER, 1992; CONRAD; POTTER, 2003; CONRAD; BERGEY, 2014; BERGEY et al., 2018).

A mediados de la década de 1970, analizó el actual diagnóstico de TDAH (entonces denominado hiperkinesis) como caso empírico emblemático de las conductas desviadas que suscitaron la intervención de la institución médica en tanto agente de control social, a través de la psiquiatría y la salud pública (CONRAD, 1975). A principios de la década siguiente empleó al TDAH para ilustrar la medicalización de la anormalidad, y amplió el análisis a otros saberes e instituciones, entre ellos las escuelas, familias y compañías farmacéuticas (CONRAD, 1982). En los '90, incluyó a la hiperactividad como ejemplo de medicalización de la infancia y estableció que ésta es un segmento etario de destacado interés a los efectos del control social, por ser a la vez depositaria de sanciones y reglamentaciones, y objeto de acciones de prevención y protección. Esto la convierte en un sector de la población con predisposición a quedar inserto en procesos de medicalización (CONRAD; SCHNEIDER, 1992). En este derrotero 
analítico, el TDAH también ha sido estudiado como diagnóstico pionero en el empleo de psicofármacos para problemas de conducta infantil (HEALY, 2002; SHORTER, 2005; MAYES, BAGWELL; ERKULWATER, 2008; MAYES; RAFALOVICH, 2007).

En lo que va del siglo XXI la medicalización ha experimentado dos procesos imbricados entre sí: de transformación y de expansión (CONRAD; BERGEY, 2014). Por un lado, el primer proceso, de transformación de la medicalización, incluye entre otros desprendimientos y afinamientos conceptuales a la farmacologización, como una corriente emergente de creciente relevancia en los estudios sociales (BELL; FIGERT, 2015; GREENE; SISMONDO, 2015; PETRYNA, LAKOFF; KLEINMAN, 2006; BIANCHI; FARAONE, 2018). La misma se define como la traducción de condiciones, capacidades y potenciales humanos en oportunidades para la realización de intervenciones farmacológicas terapéuticas o de mejoramiento, e incluye el recurso progresivo a intervenciones y respuestas farmacológicas para aspectos y problemas de la vida (ABRAHAM, 2010; CONRAD, 2013).

Por otro lado, el segundo proceso que caracteriza a la medicalización en el siglo XXI consiste en que, con la expansión de los diagnósticos y la consecuente prescripción de medicación como puntas de lanza, la misma se está volviendo global. Cuando surgió la perspectiva se enfocaba en investigaciones sobre problemáticas empíricas mayormente estadounidenses y británicas, y los análisis provenían de usinas anglosajonas. Pero paulatinamente se diversificaron los estudios y aportes en otras latitudes (BELL; FIGERT, 2015; CONRAD, 2013; CLARKE; SHIM, 2011). En América Latina existe una tradición de investigaciones al respecto, con publicaciones acerca de los estudios sociológicos dirigidos a los procesos de medicalización provenientes de la región y en sus especificidades epistemológicas (MURGUÍA; ORDORIKA; GUERRERO, 2016; MURGUÍA; ORDORIKA; LENDO, 2016).

En la década de 1990 se inició la expansión en la esfera internacional del diagnóstico de TDAH (BERGEY et al., 2018), y en el siglo XXI se identificaron algunos vehículos que explican su migración a otros puntos del globo. Conrad y Bergey (2014) describen un conjunto de vectores que 
propician la ampliación del TDAH como un diagnóstico medicalizado a escala global. Además de subrayar la importancia de saberes y actores no médicos en este proceso, y la penetración creciente del Manual de Diagnóstico y Estadísticas de los Trastornos Mentales (DSM) en el mundo (desde su primera versión en 1952, y con especial énfasis desde 1980, hasta la última versión, el DSM5, de 2013), destacan el crecimiento en el consumo de medicación para el TDAH en un amplio rango de países, y la pertinencia de documentar las variabilidades nacionales para una mejor comprensión de la propagación, implementación e interpretación del TDAH fronteras afuera de Estados Unidos.

En cuanto a la medicalización en el siglo XXI, entonces, la terapéutica medicamentosa del TDAH es expresiva tanto de la profundización de la tendencia hacia la farmacologización, como de la globalización de la misma, a la vez que resulta ilustrativo para el estudio del papel que cumplen diferentes saberes en tales procesos. En este proceso de farmacologización, las creencias, imaginarios y representaciones ostentan una eficacia simbólica que contribuye a su reproducción y profundización (JENKINS, 2011).

Por una parte, el metilfenidato se considera la droga de primera elección, y es el psicoestimulante más frecuentemente prescripto para el tratamiento del TDAH (BARKLEY, 2006; SINGH, 2008; MAYES, BAGWELL; ERKULWATER, 2008). Esto redunda sostenidamente en altísimos beneficios para la industria farmacéutica (CONRAD, 2005; WHITAKER, 2017). A esto se añade que la última versión del DSM hizo modificaciones en la tipificación del TDAH. Dejó de caracterizar el cuadro como un trastorno de inicio en la infancia, adolescencia y juventud, y lo clasificó como un trastorno del neurodesarrollo, lo que habilita el diagnóstico y tratamiento expandidos hacia edades adultas (APA, 2013; BIANCHI, 2016).

Por otra parte, el TDAH ha pasado de asociarse en sus inicios a una condición mayormente infantil (CONRAD; POTTER, 2003; CDC, 2018) predominantemente al interior de Estados Unidos (FARAONE et al., 2003), a configurarse como diagnóstico en creciente incremento en adultos (CONRAD; POTTER, 2003; BERGEY et al., 2018) y en ampliación hacia todo el globo (POLANCYK et al., 2007; CONRAD; BERGEY, 2014), con estudios que documentaron el proceso de arribo y consolidación del diagnóstico y del tratamiento farmacológico consecuente 
en los cinco continentes (BERGEY et al., 2018), y específicamente en el sur de América Latina (FARAONE; BIANCHI, 2018).

El objetivo general del artículo es contribuir a una mirada panorámica del fenómeno de la medicalización del TDAH en Argentina, y poner de relieve la importancia de analizar los múltiples saberes, actores o fuerzas sociales e instituciones que actualmente están presentes en los procesos de globalización del diagnóstico de TDAH, como ejemplo paradigmático de las discusiones acerca de la diseminación de los mismos sobre las infancias. Para ello, nos interesa avanzar en un análisis del comportamiento de los fármacos desde una perspectiva de las ciencias sociales.

Más ampliamente, con el artículo nos interesa engrosar los todavía escasos estudios que aportan a la comprensión de cómo el diagnóstico y el tratamiento farmacológico del TDAH están migrando a diferentes regiones del globo, y más específicamente, cómo está posicionándose el TDAH como un problema de salud pública en el cono sur de América Latina (BIANCHI et al., 2016; BIANCHI et al., 2017; FARAONE; BIANCHI, 2018; BIANCHI et al., 2020).

Desde una perspectiva de análisis crítica de los procesos de medicalización que toma como encuadre teórico producciones académicas recientes, y como un aporte a este campo de estudios, en este artículo presentamos resultados de investigaciones conducidas en Argentina de manera ininterrumpida desde 2008 a la fecha, en las que analizamos sistemáticamente a la industria farmacéutica como uno de los actores no médicos más relevantes para la comprensión de los modos en que Argentina participa de la globalización del TDAH, con resultados de análisis de datos cuantitativos y cualitativos. Este actor social pone en juego diferentes saberes respecto de los fármacos, que pueden ser integrados en un análisis desde los procesos de farmacologización de la sociedad, y que se integran de modos no siempre armónicos con diferentes expectativas sociales, culturales y educativas, crecientemente preocupadas por aquellos nińos y niñas que no se adaptan a cánones y expectativas tradicionales.

A fin de dar curso a los objetivos arriba planteados, efectuamos una sistematización de información para la que se efectuó una relectura y profundización del encuadre teórico en claves no exploradas previamente, lo que dio lugar a nuevas líneas de análisis, formuladas por primera vez 
para este artículo, en relación a las dos facetas de la medicalización en el siglo XXI que nos interesa analizar.

En lo atinente a la concepción del artículo, la metodología elaborada sigue una estrategia en dos planos, diseńada para este escrito. Una parte de las fuentes fueron obtenidas en investigaciones precedentes, pero fueron reconsideradas y reprocesadas en función del objetivo e hipótesis del artículo, de manera que los desarrollos aquí planteados constituyen resultados noveles y articulados con las perspectivas y corrientes de estudio en la que se sustentan. Otra parte de los datos, que completan las series de información sobre importación de metilfenidato entre 2013 y 2016, y de dispensación de metilfenidato y atomoxetina entre 2006 y 2016, fueron obtenidos y procesados, y son presentados también, por vez primera para este trabajo.

Por último, y dado que asumimos a la terapéutica medicamentosa del TDAH como un fenómeno complejo, con diferentes saberes y actores sociales en tensión, y que no se encuentra saldado sino en curso, entendemos que los resultados del artículo no configuran una lectura consolidada, sino que son útiles para establecer tendencias y mapear procesos que involucren múltiples saberes, con también múltiples dimensiones, que expresan sus coincidencias y disidencias respecto del diagnóstico y terapéutica del TDAH, a la vez que vehiculizan representaciones, imaginarios y expectativas culturales que se asignan a los y las niños y niñas. Por ello, también consideramos que estos análisis deben ser integrados y triangulados con otras fuentes y documentos para una apreciación del problema que pueda captar los matices antedichos.

\section{Medicalización y TDAH en el siglo XXI: nuevos procesos y perspectivas}

Como anticipamos, tanto la transformación como la expansión de la medicalización son procesos de reciente configuración. A la vez, el inicio de las publicaciones de estudios académicos que documentan estos fenómenos y sus efectos es coincidente con el cambio de siglo.

Específicamente, estas dos aristas de la medicalización en el siglo XXI vienen siendo abordadas desde dos perspectivas que se han consolidado también desde hace pocos años, y que están íntimamente vinculadas a la 
medicalización de la sociedad. Por una parte, la farmacologización de la sociedad y por otro la sociología del diagnóstico

\section{I Farmacologización y transformación de la medicalización}

La industria farmacéutica transnacional se ha posicionado en la actualidad como un actor social que genera informaciones, y condensa conocimientos y saberes, y está dotado de inobjetable preeminencia en los procesos de medicalización, a la vez que se ha erigido como potencia mundial, tanto en las economías globalizadas como en algunas economías nacionales (BUSFIELD, 2006).

En este contexto, la farmacologización surge como un desprendimiento y afinamiento de los estudios de la medicalización y biomedicalización (BELL; FIGERT, 2015). Se ha tornado en una perspectiva de análisis en sí misma debido, entre otros factores, a la antedicha creciente relevancia de las industrias farmacéuticas transnacionales y de las innovaciones en genética (GREENE; SISMONDO, 2015). Su emergencia además expresa la insuficiencia de la noción de medicalización para dar acabada cuenta de los enlaces a escala global que se promueven entre la medicina, la industria farmacéutica y el campo de la ciencia y la tecnología. Así, la farmacologización como concepto ha sido definido como la traducción de condiciones, capacidades y potenciales humanos en oportunidades para la realización de intervenciones farmacológicas terapéuticas o de mejoramiento, sea de parte de los médicos, de los pacientes o de ambos (ABRAHAM, 2010).

Al igual que la medicalización, la farmacologización es un proceso, y está dotada de una cierta reversibilidad. Conrad (2013) define a esta característica como bidireccionalidad, y la misma consiste en que, así como la farmacologización puede avanzar sobre los procesos sociales más amplios, es también posible una des-farmacologización. Sin embargo, los casos empíricos en los que esta tendencia se verifica son marginales respecto de la tendencia opuesta que se ha documentado, hacia la farmacologización de la vida.

Si la abordamos como la utilización de terapéuticas o respuestas medicamentosas para enfrentar problemas de la vida, la farmacologización 
se revela como un proceso que, aunque está relacionado con el campo de lo médico y de lo medicalizable, también lo excede. Por eso esta perspectiva más reciente de estudios tiene el valor de ofrecer herramientas que permiten distinguir algunas características que la noción de medicalización no trabaja específicamente. La farmacologización, en resumen, es un proceso sociotécnico complejo, dinámico y múltiple, y estas características se prolongan en los actores que involucra, y que incluyen diversos de clínicos, pacientes, consumidores y organismos de regulación, que participan de la configuración de la industria farmacéutica. Una configuración cuyos contornos y efectos todavía no han terminado de producirse (WILLIAMS, MARTIN; GABE, 2011).

Greene y Sismondo (2015, p. 6-7) aportan que: “[...] los fármacos se convirtieron en significantes sociales y culturales cuyos significados no están completamente controlados por quienes los prescriben, ni por los marcos legales y regulatorios que gobiernan el consumo farmacológico”4

La dimensión de lo social y cultural en el análisis del fármaco se vuelve especialmente relevante en sociedades en las que el consumo no sólo se encuentra naturalizado, sino que opera como estructurante del yo, como así también los estudios que reconocen la ligazón del fármaco con otras nociones tales como la idea de yo, el mundo social, la comunidad e incluso la Nación (JENKINS, 2011).

\subsection{Sociología del diagnóstico y expansión de la medicalización}

La sociología del diagnóstico es la resultante de la intersección de diferentes campos y perspectivas de análisis social, entre ellas la sociología médica, la medicalización, la historia de la medicina y la teoría de la enfermedad. Sin embargo, se ha consolidado como una subdisciplina con peso propio en la que convergen objetos de estudio, tradiciones de análisis, conceptos, métodos y problemáticas empíricas que requieren una aproximación y reflexión específicas (JUTEL, 2009, 2015).

4 Pills become social and cultural signifiers whose meanings are not full controlled by prescribers or by the legal and regulatory frameworks that govern pharmaceutical consumption. 
Desde las ciencias sociales, el diagnóstico ha sido definido como la herramienta clasificatoria de la medicina que orienta la atención médica, organiza el cuadro clínico, jerarquiza las opciones terapéuticas (entre ellas, la terapéutica farmacológica), en ocasiones busca predecir resultados futuros, y expone un marco explicativo. Pero además el diagnóstico encuadra la formación e intervención profesional. Como el diagnóstico también define qué profesional es responsable de cada cuestión, también opera como estructurador y diferenciador de las relaciones entre legos, profesionales y especialistas dentro de cada profesión (JUTEL, 2011a, 2011b).

El diagnóstico abre la puerta a reflexiones acerca de la enfermedad, la salud, el sufrimiento, como así también a distintas aristas que incumben tanto a los conocimientos como a las prácticas. El diagnóstico no es ajeno a la dimensión ideológica, por lo que contribuye a establecer distintas modalidades de normalización y de conformación de subjetividades (JUTEL, 2011a, 2011b).

El diagnóstico, entonces, es central para la medicina y para comprender los modos en que configura el orden social (JUTEL, 2009, 2011a, 2011b). Por eso el diagnóstico es más que un procedimiento para establecer la etiología de un conjunto de síntomas; cumple toda otra serie de funciones. Entre ellas, como insumo para las estadísticas y archivos de instituciones oficiales y de tipo clínico; para la incorporación o rechazo de un paciente a investigaciones, estudios y ensayos; en las alternativas de tratamientos e intervenciones; en estimaciones o predicciones respecto de cuadros futuros; en la posibilidad de calificar o no para trabajos, seguros o pensiones; para la elaboración de políticas públicas epidemiológicas y de salud; para la asignación o recorte de recursos; y para el diseño y aplicación de campañas de marketing farmacéutico, entre otras tantas (ROSE; ABI-RASCHED, 2013).

Estas funciones responden a dimensiones, normas dinámicas, lógicas y efectos disonantes y en tensión entre sí, y por eso el diagnóstico puede entenderse como una arena de conflictos en los que interactúan actores y fuerzas sociales, discursos, dispositivos, saberes, prácticas y tecnologías.

Específicamente en el campo de la salud mental, el diagnóstico está incidido además por cuestiones que no son clínicas ni psiquiátricas, sino sociales y cotidianas, pero a las que se les otorga relevancia diagnóstica, entre 
ellas los antecedentes educativos, las actividades sociales, detalles de la vida afectiva y familiar y la capacidad de administrar el dinero (ROSE, 1996).

En el estudio de la expansión de la medicalización, y tomando en cuenta los aportes de la sociología del diagnóstico, una pieza ineludible para la reflexión y análisis de los diagnósticos en salud mental la constituyen los manuales de clasificación, y específicamente en el caso del TDAH, el DSM.

Desde 1952, el DSM es publicado por una asociación privada de psiquiatras estadounidenses, la American Psychiatric Association (APA), y ha suscitado fuertes controversias y críticas en relación al diagnóstico en salud mental, y al concepto de trastorno mental en particular (FARAONE, 2013; BIANCHI, 2014b; BIANCHI, 2016). Las diferentes versiones del DSM describen una curva que comprende desde la psicología con referencias psicoanalíticas en las dos primeras versiones, pasando por la psiquiatría biológica y el empirismo baconiano de la versión III, en 1980 (BIANCHI, 2014b), luego la expansión a escala global de la versión IV, de 1994, hasta la definición nueva de trastorno mental de la versión 5, en 2013 (BIANCHI, 2016).

La expansión de la psiquiatría estadounidense a escala global fue posible entre otras razones, por el uso cada vez más extendido del DSM (CONRAD; BERGEY, 2014, 2014; REED et al., 2011).

En particular, el DSM-5 ha incorporado nuevas nomenclaturas y nuevos criterios diagnósticos. La profundización del modelo dimensional en esta versión del manual trajo como consecuencia una ampliación de los diagnósticos. En el caso del TDAH, esta ampliación diagnóstica se hace patente en su extensión como diagnóstico en adultos, y esto supone una transformación en relación a tipificaciones previas, que lo caracterizaban como un diagnóstico preeminentemente infantil (BIANCHI, 2016). Esta ampliación marca la pauta de cómo las categorías diagnósticas que están inscriptas en los procesos de medicalización son dinámicas, y pueden expandirse e incluir otros segmentos poblacionales (CONRAD; POTTER, 2003).

\section{Metodología}

El diseño metodológico consiste en una estrategia en dos planos, con análisis de fuentes cuantitativas, que se complementan, amplían y profundizan con fuentes cualitativas. 
Parte de las fuentes se obtuvieron en investigaciones precedentes, pero fueron reconsideradas y reprocesadas en función del objetivo e hipótesis del artículo, de modo que los desarrollos constituyen resultados noveles, articulados con las perspectivas y corrientes de estudio en las que se sustentan. Otra parte de los datos que completan las series de información sobre importación de metilfenidato entre 2013 y 2016, y de dispensación de metilfenidato y atomoxetina entre 2006 y 2016 se solicitaron a los organismos pertinentes, procesaron y presentan por vez primera aquí.

En cuanto a las fuentes cuantitativas, se realiza un análisis estadístico descriptivo (HERNANDEZ SAMPIERI; FERNANDEZ COLLADO; BAPTISTA, 1998; CEA D’ANCONA, 1996) de datos provenientes de diferentes organismos nacionales $\mathrm{e}$ internacionales $\mathrm{y}$ asociaciones profesionales:

1. Consumo mundial de metilfenidato, en millones de S-DDD (sigla en inglés de daily doses for statistical purposes, dosis diarias definidas con fines estadísticos) y fabricación mundial de metilfenidato en toneladas, ambos para el período 1990-2013. Los datos provienen de la Junta Internacional de Estupefacientes (JIFE, o INCB por la sigla en inglés de Internacional Narcotics Control Board).

2. Importación anual de metilfenidato como monodroga en Argentina, en kilos, para el período 2003-2016. La Administración Nacional de Medicamentos, Alimentos y Tecnología Médica (ANMAT), a través de su Departamento de Psicotrópicos y Estupefacientes, proveyó la información para los períodos 2005-2008 y 2011-2016. Los datos para el resto de los años de la serie se obtuvieron de otras fuentes secundarias, periodísticas y de literatura específica sobre el tema, que detallamos más adelante.

3. Datos de cantidad de unidades dispensadas (expresadas en envases), tanto de metilfenidato como de atomoxetina en todas sus presentaciones comerciales, a nivel nacional. Esta información está mensualizada, y corresponde a dos períodos de tiempo: 2005-2010 y 2012-2016. Estos datos los proveyó la Confederación Farmacéutica Argentina (COFA), a través de su Observatorio de Salud, Medicamentos y Sociedad. Al momento de solicitar los datos, la COFA no disponía de información del año 2011.

Los datos cuantitativos se procesaron e integraron a una lectura de fuentes cualitativas, consistentes en 84 entrevistas semiestructuradas, 
individuales y grupales, realizadas entre 2007 y 2017 (AUTOR1, OTRO, OTRO, AUTOR3, AUTOR1, OTRO, ET AL, 2008;; AUTOR1, 2012; 2014a; AUTOR2 \& AUTOR1, 2008). Todas siguieron un criterio muestral intencional no probabilístico, orientado a profesionales con actividad clínica, docente y de investigación en ámbitos públicos, de la seguridad social y del subsector privado.

Durante los diez años de trabajo de campo, se realizaron cuatro tandas de entrevistas a profesionales de la salud y la educación que ejercían su actividad en la Ciudad de Buenos Aires y Área Metropolitana. Adicionalmente entrevistamos profesionales farmacéuticos y otros informantes clave. Durante el período 2007-2008 se amplió la toma de entrevistas a nivel nacional, y seleccionamos tres jurisdicciones (Salta, Tierra del Fuego y Corrientes) como puntos muestrales que representaban regiones del país con diferentes intensidades de dispensación de metilfenidato y atomoxetina en relación a la población de entre 5 y 19 ańos (FARAONE $e t$ al., 2008). Para el artículo trabajamos cuatro dimensiones de las entrevistas que profundizaron la lectura de los datos cuantitativos: 1) nosología, 2) quiénes son diagnosticados, 3) terapéuticas y 4) psicofármacos. El cuadro 1 resume las características de la muestra de entrevistas.

La Tabla 1 resume las características de la muestra de entrevistas semiestructuradas.

El entramado teórico, el diseño metodológico y las fuentes llevan a postular dos hipótesis: una acerca de tendencias nacionales que son similares a las globales, y otra relacionada a aspectos con particularidades y matices propios de Argentina.

La hipótesis que denominamos de la consonancia con procesos globales consiste en sostener que, al igual que en el resto del mundo, en Argentina el metilfenidato describe una tendencia alcista, tanto en la importación de la monodroga, como en la dispensación del producto manufacturado. Esta hipótesis permitiría también asumir que el metilfenidato, igual que en otros países, sería la droga de mayor venta en Argentina para el tratamiento medicamentoso del TDAH, sin desconocer que en el país también se comercializa la atomoxetina para el tratamiento de dicho diagnóstico.

Luego, la hipótesis que caracterizamos como aquella disonante con procesos descriptos globalmente (CONRAD; POTTER, 2003; BERGEY et al., 2018), refiere a que en Argentina todavía la tendencia de dispensación 
de metilfenidato y atomoxetina se asociaría, aunque no excluyente ni directamente, a población en edad escolar.

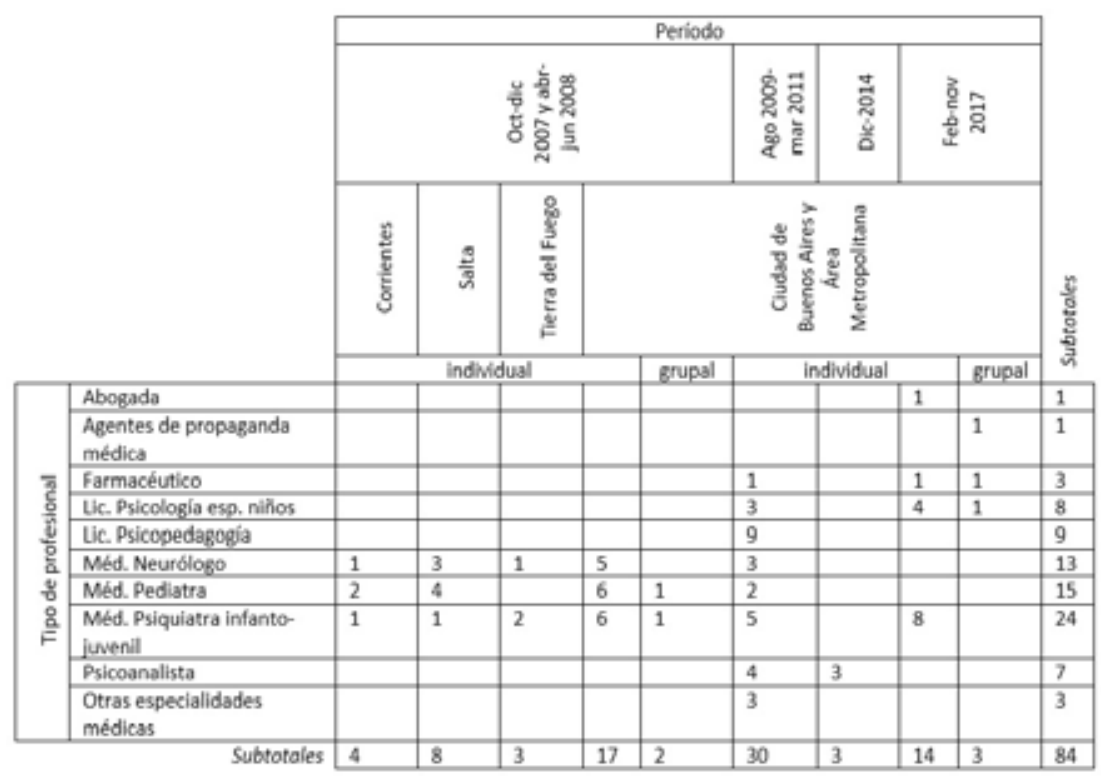

Tabla I - Características de la muestra de entrevistas 2007-2017. Fuente: Elaboración propia en base a entrevistas realizadas.

La medicalización no alude a procesos unívocos, homogéneos o generales (BIANCHI et al., 2016), y tampoco los resultados a los que se arriba cuando se los investiga. Por eso, esta hipótesis no descarta la existencia de tratamientos en adultos, como tampoco asume que la totalidad de la dispensación de metilfenidato y atomoxetina se destinan íntegramente al tratamiento medicamentoso del TDAH. En cambio, se orienta a presentar algunos elementos que sugieren que la problemática del TDAH en Argentina se distingue de tendencias analizadas en otros países, en los cuales se ha documentado que el cuadro ya se expandió y afianzó también en aquel segmento poblacional.

Asimismo, esperamos que estas primeras aproximaciones e hipótesis puedan profundizarse y contrastarse en futuras investigaciones, a la vez que 
sienten las bases para análisis que permitan comparar esta línea de estudios con las realidades fenoménicas de otros países y regiones.

Las investigaciones se ajustaron a normativas y resguardos éticos vigentes para los estudios sobre salud humana. Los datos se obtuvieron atendiendo a la Resolución del Ministerio de Educación, Ciencia y Tecnología $n^{\circ}$ 2857/2006 y a la Resolución 1480/2011 del Ministerio de Salud. Se utilizaron consentimientos informados para la realización de las entrevistas. Los mismos se documentaron a través de formularios escritos y fechados que se entregaron a los involucrados, explicitando que la información obtenida en la entrevista revestiría carácter confidencial y anónimo. Los planes de trabajo de las investigaciones y consentimientos informados en el marco de los cuales fue elaborado el artículo fueron evaluados y avalados por el Comité de Ética del Instituto de Investigaciones Gino Germani de la Facultad de Ciencias Sociales de la Universidad de Buenos Aires, en el que ambas autoras somos investigadoras. El Instituto no codifica la aprobación de los proyectos.

Por último, declaramos no tener conflictos de intereses políticos y/o financieros asociados a fuentes de financiamiento, patentes o propiedad, provisión de materiales y/o bienes y equipamientos.

\section{Discusión y resultados}

\section{I Metilfenidato y atomoxetina en Argentina}

Una persistente dificultad en las investigaciones sobre TDAH es obtener datos numéricos oficiales. Argentina no publica información estadística oficial sobre estimaciones de prevalencia de diagnósticos, ni de prescripción o consumo de fármacos relacionados. Por ello, la incorporación de información estadística a nuestras investigaciones es fragmentada, parcial y asistemática.

No obstante sus limitaciones los datos recabados permitieron establecer tendencias y realizar mapeos amplios sobre cómo se está dando el panorama a este respecto. También auxiliaron para realizar algunas hipótesis interpretativas, lo que consideramos relevante para la comprensión global de la problemática y su expresión en el país. Por último, y dado el antedicho carácter fragmentado, parcial y asistemático de los datos cuantitativos, 
encuadramos e integramos los mismos en un análisis que incluyó también otras fuentes de corte cualitativo obtenidas en las investigaciones.

En Argentina se comercializan dos fármacos para el tratamiento medicamentoso del TDAH, el metilfenidato y la atomoxetina.

El metilfenidato es un estimulante del sistema nervioso central con propiedades farmacológicas similares a las anfetaminas. En la clasificación de los psicofármacos, integra el grupo de los psicoanalépticos, que comprenden a los psicoestimulantes o estimulantes de la vigilia, y a los antidepresivos o estimulantes del humor. El metilfenidato pertenece al primer grupo. Al cierre del artículo, en Argentina lo comercializan cuatro laboratorios, en 16 presentaciones y ocho concentraciones diferentes, bajo la forma de comprimidos, cápsulas de liberación modificada y comprimidos recubiertos de liberación prolongada.

Su distribución, al igual que en otros países, requiere de una serie de controles y requisitos. En entrevistas a profesionales e informantes clave nos refirieron que tanto el profesional médico que lo indica, como el farmacéutico que lo dispensa deben estar registrados en un listado oficial habilitante. Existen también protocolos de trazabilidad de envases y recetas. Además, las farmacias donde se dispensa deben cumplir requerimientos de seguridad en relación a condiciones de almacenamiento.

Por otrolado, la atomoxetina es un agente simpaticomimético, inhibidor selectivo de la recaptación del neurotransmisor norepinefrina, por lo que incrementa su actividad. A diferencia del metilfenidato, no se considera un estimulante, lo que supone controles y requisitos menos estrictos. En Argentina, al cierre del artículo la comercializan dos laboratorios, bajo la forma de cápsulas, en seis presentaciones y seis concentraciones diferentes.

En los prospectos de las diferentes marcas de ambos principios activos comercializadas en el país que figuran en el vademécum online, se detalla que los fármacos están indicados exclusivamente para el tratamiento del TDAH (www.alfabeta.net). Además, se aclara que la administración de ambos fármacos debe formar parte de un programa integral de tratamiento del TDAH, como coadyuvante, y que dicho programa debe incluir otras medidas además de las farmacológicas, entre ellas sociales, psicológicas y educacionales. En otros artículos (BIANCHI et al., 2017; BIANCHI; 
FARAONE, 2015) analizamos el impacto de los grupos de profesionales críticos de la terapéutica medicamentosa en Argentina, y cómo sus reclamos derivaron en modificaciones y especificaciones en los prospectos y normativas relacionadas con la prescripción y dispensación de medicación que se indica como tratamiento psicofarmacológico de primera línea para el TDAH en el país.

Además, los profesionales e informantes nos refirieron la vinculación observada tanto en su experiencia clínica, como en diversos ateneos y congresos, respecto del incremento del diagnóstico, y el aumento de la indicación de estos psicofármacos como fenómenos concomitantes. Por todo ello, proponemos en este artículo una asociación, en los términos de una co-ocurrencia o covarianza, entre la dispensación de metilfenidato y atomoxetina, y el diagnóstico del TDAH.

A continuación, presentamos los resultados en función de las dos hipótesis planteadas.

\subsection{Hipótesis I: La consonancia entre tendencias mundiales y nacionales, y el predominio del metilfenidato}

Retomando la discusión planteada, desde la primera investigación realizada (FARAONE et al., 2008), y en cada investigación subsiguiente (BIANCHI, 2012, 2014a, BIANCHI; FARAONE, 2015; FARAONE; BIANCHI,), constatamos las reiteradas referencias de parte de profesionales entrevistados acerca de que en Argentina el metilfenidato es la droga de primera elección para el tratamiento medicamentoso del TDAH, y también la de mayor venta en este país. Estas consideraciones son consonantes con estudios académicos internacionales (SINGH, 2008; SINGH et al., 2013; BIANCHI et al., 2016; BIANCHI et al., 2017) e informes de la JIFE que también utilizamos como fuente de información cuantitativa (JIFE, 1995, 2006, 2012, 2014, 2015).

Aunque la comercialización de este psicofármaco se remonta a la década del 1950 en Estados Unidos (HEALY, 2002; BERGEY et al., 2018; BARKLEY, 2006) está documentado que la asociación cada vez más directa con el TDAH se relaciona con el aumento internacional en la producción y el consumo del psicoestimulante a partir de la década de 1990 (JIFE, 1995, 2006). 
En 2012, la JIFE señaló que la fabricación mundial de metilfenidato se había más que decuplicado entre 1992 y 2011 , ya que pasó de 4,2toneladas en 1992 a 45,2 toneladas en 2011, y en 2009 superó la cifra mundial combinada de fabricación de todas las anfetaminas. El consumo mundial aumentó durante el mismo período de 4,2 toneladas a 51 toneladas. El informe también detalla que la cantidad de S-DDD aumentó de 139 millones a 1.500 millones (JIFE, 2012).

En 2014 la JIFE publicó la serie del consumo mundial de metilfenidato para el período 1990 a 2013 (JIFE, 2014), y al ańo siguiente la serie de fabricación mundial para el mismo período (JIFE, 2015). Ambos informes dan cuenta de un acompasamiento en el incremento.

Según la JIFE, durante la década de 1980 el empleo de metilfenidato era limitado y sus niveles se mantenían estables. A principios de la década de 1990 el aumento empezó a ser notorio: en 1994 el uso mundial de esta sustancia superó en más de cinco veces el nivel de consumo registrado a principios de la década anterior (JIFE, 2014). Esa evolución se debió principalmente al incremento del consumo en Estados Unidos, aunque también se observó con menor intensidad en otros países. Desde entonces, el consumo mundial aumentó de manera constante. En 2013 se alcanzó el récord, de 71,8 toneladas (2.400 millones de S-DDD) (JIFE, 2014). Sin incluir los posibles usos no terapéuticos de la sustancia, y considerando lo hasta aquí analizado acerca de las investigaciones internacionales, se podría interpretar que, si su prescripción se realiza mayoritariamente para el tratamiento del TDAH, estaría indicando un mayor número de diagnósticos de este trastorno.

El informe de 2015 de la JIFE ilustra el incremento gradual pero sostenido de la fabricación mundial de metilfenidato entre 1990 y 2013. Ese último año la producción mundial alcanzó aproximadamente 72 toneladas. El informe consigna que más del $70 \%$ de las importaciones mundiales de metilfenidato se llevaron a cabo en siete países de Europa y América: Suiza, Alemania, España, Canadá, Brasil, los Países Bajos y el Reino Unido (JIFE, 2015).

Como señalamos, en Argentina diferentes profesionales entrevistados y múltiples artículos periodísticos relevados en el marco de nuestras investigaciones refieren un incremento sostenido en la prescripción y consumo del metilfenidato en el siglo XXI, dato que asocian con el incremento en el diagnóstico de TDAH en el país. Los entrevistados no 
fundamentan esta asociación en indicadores estadístico-epidemiológicos (algo que es consistente con la ausencia de publicaciones oficiales) sino que, argumentan, se trata una verificación permanente en el consultorio, y en la práctica clínica en salud mental infantil y neurología infantil (BIANCHI; FARAONE, 2015).

Para comparar la información global con la de Argentina, utilizamos dos tipos de datos. Por un lado, la cantidad de kilos de metilfenidato importados por ańo, en base a datos provistos por ANMAT. Argentina no produce metilfenidato, aunque sí importa la monodroga y elabora el principio activo en distintos laboratorios. Luego se comercializa internamente y se exporta a algunos países limítrofes. Sin embargo, informantes clave del campo farmacológico han referido que también, y paralelamente, algunos laboratorios importan metilfenidato ya elaborado de Brasil. De manera que, aunque este dato da una idea del quantum de monodroga ingresada al país, no representa la totalidad del metilfenidato disponible para consumo interno, ya que existen varios circuitos de importación y exportación del producto terminado.

Por otro lado, tomamos de la COFA la cantidad de unidades de metilfenidato dispensadas (entendidas como envases de las diferentes presentaciones). Dada la dispersión de dosis y formas de liberación que pueden combinarse en cada tratamiento individual, este dato no es asimilable a perfiles de consumo, pero brinda un panorama acerca de las cantidades de fármaco dispensado.

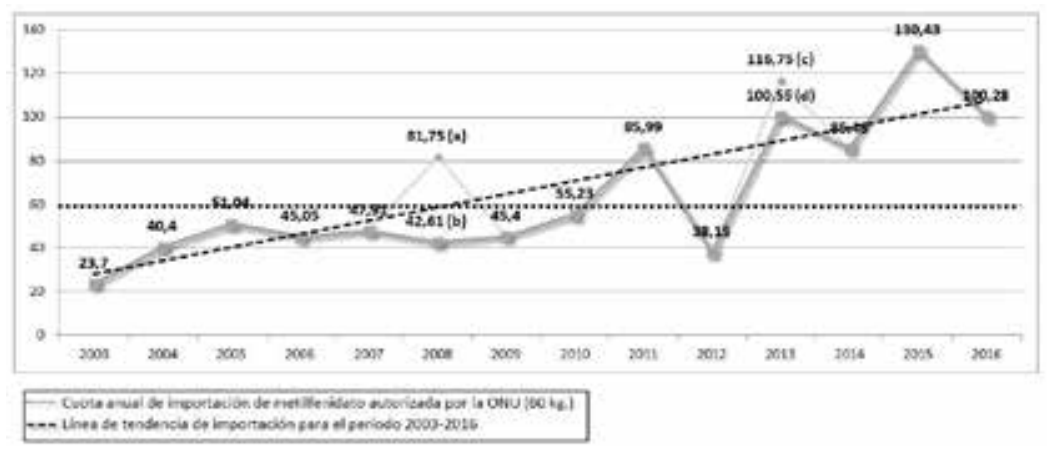

Gráfico I - Kilogramos de metilfenidato (como monodroga) importando anualmente en Argentina (2003-2016)

Fuentes: Elaboración propia en base a fuentes: 2003-2004 (CARBAIAL, 2007); 2005-2008 (AUTORI, OTRO, OTRO, AUTOR3, AUTORI, OTRO, ET AL, 2008); 2008-20I0 (MANIOWICZ, 20II); $2011-2016$ (ANMAT). 
La información arroja que en el período considerado la tendencia de importación de metilfenidato en Argentina para su comercialización es marcadamente alcista, una tendencia consonante con la mundial. En el gráfico se registran discrepancias en los valores señalados por las distintas fuentes para los años 2008 y 2013. Así, Faraone et al. (2008) refieren para 2008 un valor por encima de los 80 kilos (a) y Maniowicz (2011) refiere un valor sensiblemente menor (b). Para 2013, datos obtenidos de ANMAT en diferentes momentos, arrojan una diferencia mucho menor entre sí (c) y (d), aunque igualmente alta.

Estas cifras son significativas, en tanto la cuota anual de importación acordada por la Administración de Alimentos y Fármacos de Estados Unidos, (FDA) con la Organización de las Naciones Unidas está establecida para Argentina en 60 kilogramos (CARBAJAL, 2007) y los datos recabados exceden decididamente este tope desde 2008, con excepción de 2012. Asimismo, aunque los valores de importación oscilan cada año, y desde 2010 en adelante estos altibajos son más pronunciados, a los efectos de señalar una tendencia hay congruencia en un incremento sostenido en la importación del metilfenidato. De modo que, aunque no es posible asumir que todo el metilfenidato que se elabora en el país a partir de la monodroga importada se consume fronteras adentro, tampoco se puede desestimar que exista una relación entre la tendencia alcista y un incremento del consumo interno.

Para estimar el incremento de la cantidad de kilos de metilfenidato importados en el período analizado, se puede considerar la información de 2003 contra la resultante del promedio entre 2015 y 2016, cifra que permite aminorar las variaciones más abruptas en los datos interanuales que se verifican desde 2010. El incremento de kilos de metilfenidato importados entre 2003 y el promedio de 2015-2016 es de 387\%, es decir un aumento de casi cuatro veces.

Esto abona a lo que sostenemos desde la investigación de 2008: que el aumento de importación de la sustancia puede obedecer a una venta efectiva creciente de los laboratorios o a una decisión de afianzar una mayor penetración en el mercado nacional y regional (FARAONE et al., 2008).

Otra información provista por la COFA es las unidades dispensadas de metilfenidato. Este no es un dato equiparable al de kilos de importación 
de monodroga, porque en este caso se trata de la droga ya elaborada por los distintos laboratorios, y empaquetada para la venta en diferentes dosis y presentaciones. Pero a fines de identificar un panorama, vale atender a su comportamiento para los períodos 2005-2010 y 2012-2016.

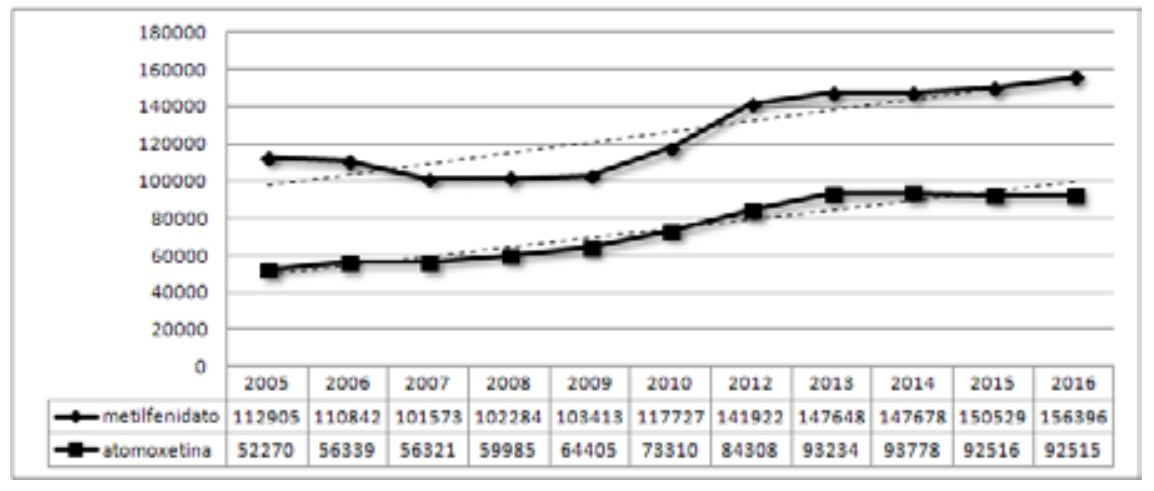

Gráfico 2 - Total de envases de atomoxetina y metilfenidato dispensados anualmente en Argentina (2005-2010 y 2012-2016)

Fuente: Elaboración propia en base a datos de la COFA (2020).

En el gráfico se observa una pequeña curva a la baja en la cantidad de unidades vendidas entre 2007 y 2010 , con un incremento del 39\% en las unidades dispensadas entre 2005 y 2016, un valor mucho menor que el incremento en la cantidad de metilfenidato importado para un período similar. Pero nuevamente, si se toma en cuenta la totalidad del período, la tendencia en los totales anuales de unidades dispensadas es alcista, una tendencia que se acentuó desde 2012.

Para ampliar el análisis de la hipótesis, nos interesa exponer algunos datos de dispensación del otro fármaco indicado exclusivamente para el TDAH en Argentina, la atomoxetina. Algunos profesionales entrevistados refirieron que esta droga ingresó al país con posterioridad al metilfenidato, en 2004 y que, como ilustra el Gráfico 2, evidencia una dispensación menor a este en unidades. La información también arroja que en 2010 se produjo un salto de unidades de atomoxetina dispensadas respecto de ańos anteriores. Esta alza se mantuvo en 2012 y 2013, y disminuyó muy levemente entre 2014 
y 2016. También en este caso, considerando todo el período la tendencia es alcista, con un incremento del 77\% entre 2005 y 2016.

Con respecto al análisis comparativo entre ambos fármacos, los datos recabados se refieren a unidades dispensadas, y no a cantidades de fármacos ni a consumo de los mismos. Las unidades de cada uno de los dos psicofármacos no pueden equipararse entre sí, una a una, dada las múltiples presentaciones, dosis y formas de liberación tanto de metilfenidato, como de atomoxetina. Sin embargo, los datos marcan un panorama de interés a efectos de conocer tendencias, y refuerza la hipótesis acerca de que el metilfenidato es la droga de primera elección, así como la de mayor dispensación en Argentina en relación al diagnóstico de TDAH.

Con los recaudos señalados el Gráfico 2 consigna que todos los años de ambos períodos considerados el metilfenidato exhibe mayor cantidad de unidades dispensadas.

El análisis se amplía al considerar las razones entre metilfenidato y atomoxetina dispensados en Argentina en los dos períodos. De los cálculos surge que los dos años posteriores a la introducción de la atomoxetina en el mercado local (2005 y 2006) la razón entre ambos fármacos era más pronunciada a favor del metilfenidato, con razones cercanas a 2 . Es decir que por cada unidad de atomoxetina se dispensaron dos (o más) de metilfenidato. En 2007 aminoró la tendencia, y desde entonces la razón no alcanza los dos puntos, si bien el metilfenidato sigue presentándose como el fármaco más dispensado.

\subsection{Hipótesis 2: La disonancia con otros países. En Argentina el diagnóstico de TDAH continúa ligada, aunque no excluyentemente, al segmento infantil y adolescente}

Esta hipótesis se sustenta en otro conjunto de datos que permiten enlazar este diagnóstico con la población escolarizada, tomando la dispensación mensual de metilfenidato y atomoxetina. Tampoco en este caso se postula una asociación directa, biunívoca o exclusiva entre ambas variables, sino antes bien, seńalamos una covarianza que no excluye la incidencia de más factores. Un aspecto documentado es la estacionalidad 
en la dispensación del metilfenidato, una tendencia reportada también en estudios brasileños (BIANCHI et al., 2016; BIANCHI et al., 2017).

Volviendo al presente estudio, agrupamos los datos en dos bloques, uno para 2005-2010 y otro para 2012-2016. En cada bloque se calculó el promedio mensual de unidades dispensadas de metilfenidato y de atomoxetina. Los datos muestran para el período 2005-2016, y con excepción de 2011, que el volumen de dispensación de ambos fármacos describe un ciclo que se mantiene alto durante la cursada, y cae significativamente en los períodos de receso escolar. Los Gráficos 3 y 4 muestran los resultados sobre metilfenidato y atomoxetina, para cada uno de los bloques antedichos.

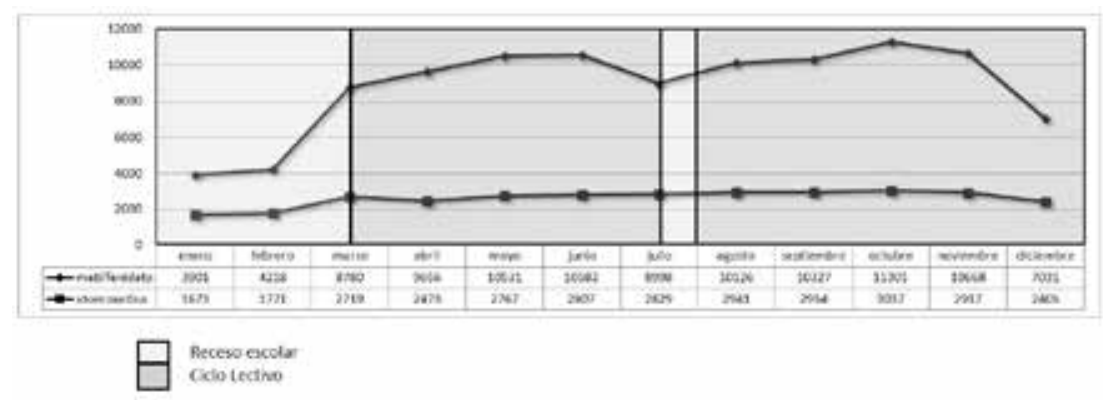

Gráfico 3 - Promedio mensual de envases de metilfenidato y atomoxetina dispensados en Argentina (2005-2010)

Fuente: Elaboración propia en base a datos de la COFA (2020).

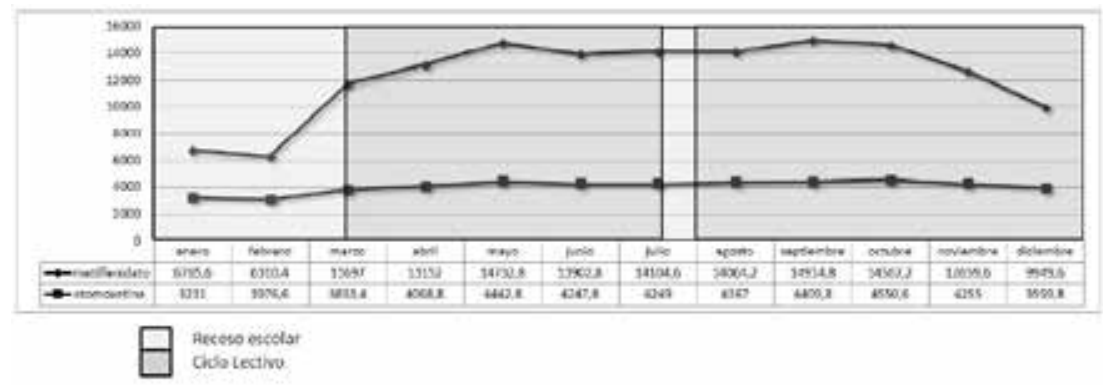

Gráfico 4 - Promedio mensual de envases de metilfenidato y atomoxetina dispensados en Argentina (2012-2016)

Fuente: Elaboración propia en base a datos de la COFA (2020). 
Para el metilfenidato, la lectura indica que los promedios de ambos períodos describen un ciclo de dispensación similar, que tiene su primer salto en marzo, coincidentemente con el inicio del ciclo lectivo, con un incremento significativo respecto del mes anterior, del $108 \%$ para el período 2005-2010, y del 85\% para el período 2012-2016.

En ambos períodos, la dispensación se mantiene en ascenso en abril, mayo y junio, y cede su tendencia al alza hacia julio, en coincidencia con el receso escolar invernal de la segunda quincena de ese mes, para retomar dicha tendencia en la segunda parte del año lectivo. Para el período 20052010 la merma en julio respecto a junio es del 15\%, y en el período 2010 2016, lo que se observa no es una merma sino un amesetamiento de la tendencia, entre junio y agosto.

El ciclo alcista se reinicia en agosto del primer período (2005-2010), y en septiembre del segundo (2012-2016), para mantenerse en ambos períodos hasta noviembre, donde inicia la baja (del 6\% en el primer período y $13 \%$ en el segundo) que se pronuncia en diciembre (del 34\% en el primer período y $21 \%$ en el segundo), en coincidencia con el inicio del receso escolar de verano.

También en el caso de la atomoxetina, ambos períodos describen un ciclo de dispensación afín, y consonante con el ciclo escolar. Sin embargo, los saltos mensuales son menos abruptos que los que se verifican para el metilfenidato, $y$ no muestran cambios tan significativos en el receso escolar invernal.

Para el período 2005 a 2010 las variaciones son respectivamente de $+54 \%$ en marzo, de $-4 \%$ en noviembre, y de $-18 \%$ para diciembre. Para el período 2012 a 2016, las variaciones son aún menos abruptas, de $+25 \%$ en marzo, de $-4 \%$ en noviembre, y de $-7 \%$ en diciembre.

En conjunto, los datos marcan una especificidad de la experiencia argentina, que evidencia un proceso de vinculación entre el diagnóstico de TDAH y la prescripción de metilfenidato más acelerado, y compactado en apenas más de una década desde 2005, lo que marca una diferencia con el más longevo proceso estadounidense, verificable desde la década de 1950 . También se observa una dispensación alta y constante de atomoxetina. Más ampliamente los datos dan la pauta del afianzamiento de Argentina como zona de producción y circulación de ambos psicofármacos. 
Una interpretación posible de los resultados es que, más allá del retiro del Manual DSM-5 del TDAH como diagnóstico de inicio en la infancia o adolescencia, en Argentina la dispensación de ambos fármacos describe una inobjetable periodicidad relacionada con el ańo lectivo, en una ciclicidad que resulta más pronunciada en el metilfenidato que en la atomoxetina.

La relevancia de postular esta hipótesis acerca de un análisis en clave de asociación de ambos datos (ciclo escolar y dispensación mensual de psicofármacos) suma elementos en virtud de lo recabado en las entrevistas a profesionales, quienes refirieron que la terapéutica medicamentosa del TDAH se orienta de acuerdo a una planificación flexible de la prescripción del psicofármaco, y se organiza de modo tal de posibilitar el cumplimiento de las actividades concretas que realiza el niño durante el día. Estas actividades varían en función de las características del ciclo escolar (doble escolaridad, días de la semana o fin de semana, época lectiva o vacacional, cursada con otros niños o clases individuales con psicopedagoga, entre otros), y de la gravedad del cuadro. Así, enfatizan que la periodicidad y la dosis en el suministro se adecuan a las variabilidades individuales de cada caso bajo tratamiento (BIANCHI, 2014a).

\section{Conclusiones}

La primera conclusión se relaciona con que, a pesar de la fragmentariedad, asistematicidad y parcialidad delainformación relacionada con el metilfenidato y la atomoxetina en Argentina, los datos cuantitativos y su articulación con datos cualitativos permiten aproximaciones y una comprensión más acabada de estos procesos. Por eso, la información que sí se logró obtener debió ser procesada y analizada tomando en consideración ciertos recaudos formales, y también ciertos postulados investigativos, consistentes en triangular la información de corte cuantitativo con resultados de fuentes cualitativas, como entrevistas y artículos periodísticos, que organizan y dan coherencia a los datos numéricos.

En segundo lugar, los resultados del procesamiento de datos del período 2005 a 2016 describen un panorama general con tendencias alcistas tanto en el caso del metilfenidato como en la atomoxetina. Para el metilfenidato, y a nivel nacional, se verificó un alza tanto en los kilos de monodroga importada 
anualmente, como en las unidades dispensadas mensualmente. Ambos datos dan cuenta de dinámicas diferentes del mercado farmacológico local, y de diferentes instancias del proceso de producción y comercialización del producto, por lo que no pueden combinarse sus resultados. Sin embargo, se documentó en ambos casos una misma tendencia al alza, pronunciada y persistente durante los períodos analizados. Para la dispensación de atomoxetina también se verificó una tendencia alcista a nivel nacional, menos abrupta que en el caso del metilfenidato, pero igualmente sostenida.

En tercer lugar, si bien los resultados no apuntan a descartar que se diagnostiquen adultos por TDAH en el país, la estacionalidad en la dispensación de metilfenidato y atomoxetina, y su covarianza con el ańo lectivo y sus recesos estival e invernal, es un dato que llevaría a considerar que sí existe una relación marcada, aunque no puede concluirse que esta relación sea exclusiva, con población en edad escolar, y opera como indicador de un cuadro que en el país continúa ligado considerablemente al segmento poblacional infantil y adolescente.

Por último, destacamos que no pueden asumirse los resultados como conclusiones taxativas, ni en términos de asociación directa, exclusiva, determinista ni biunívoca de las variables, sino bajo la forma de una covarianza. En esta línea, y si ampliamos el análisis de los resultados con el conjunto de información de corte cualitativo que deriva de nuestras investigaciones, y en función del conjunto de materiales analizados, podemos apoyar la hipótesis de que existe un componente de población en edad escolar en la dispensación de estos fármacos, lo que refuerza el planteo realizado en nuestros estudios y los de otros investigadores antes citados, sobre la necesidad y relevancia de investigar procesos de diagnóstico y tratamiento de TDAH en niñas, niños y adolescentes en el país.

\section{Referencias}

ABRAHAM, J. Pharmaceuticalization of society in context: Theoretical, empirical and Health Dimensions, Sociology, v. 4, n. 44, p. 603-622, 2010.

AMERICAN PSYCHIATRIC ASSOCIATION (APA). Diagnostic and Statistical Manual of Mental Disorders, Fifth Edition. Washington: APA, 2013.

ADMINISTRACIÓN NACIONAL DE MEDICAMENTOS, ALIMENTOS Y TECNOLOGÍA MÉDICA (ANMAT). 
Medicalización del TDAH en Argentina. Reflexiones sobre tendencias globales y especificidades locales a través del estudio del metilfenidato y la atomoxetina | Bianchi, Eugenia Faraone, Silvia A. Torricelli, Flavia C.

BARKLEY, R. Attention-Deficit Hyperactivity Disorder. A handbook for diagnosis and treatment. New York: The Guilford Press, 2006.

BELL, S.; FIGERT, A. (ed.). Reimagining (bio)medicalization, pharmaceuticals and genetics. Old critiques and new engagements. New York: Routledge, 2015.

BERGEY, M.; FILIPE, A.; CONRAD, P.; SINGH, I. (org.). Global Perspectives on ADHD: Social Dimensions of Diagnosis and Treatment in 16 Countries. Baltimore: Johns Hopkins University Press, 2018.

BIANCHI, E. ADHD y discursos de la salud sobre la infancia en el AMBA (1994-2011). Configuraciones discursivas y efectos de poder. Maestría en Investigación en Ciencias Sociales, Universidad de Buenos Aires, 2012.

BIANCHI, E. Gubernamentalidad, tecnologías y disputas. Una genealogía de los saberes profesionales acerca del ADHD en la infancia (1994-2012). Doctorado en Ciencias Sociales, Universidad de Buenos Aires, 2014a.

BIANCHI, E. Todo tiene un principio... y en el principio fue el DSM-III. El desbloqueo epistemológico y tecnológico de la psiquiatría biológica estadounidense. Culturas Psi, n. 3, p. 87-114, 2014b.

BIANCHI, E. Diagnósticos psiquiátricos infantiles, biomedicalización y DSM: ‘hacia una nueva (a)normalidad? Revista Latinoamericana de Ciencias Sociales, Niñez y Juventud, n. 14, v. 1, p. 417-430, 2016.

BIANCHI, E.; FARAONE, S. El Trastorno por Déficit de Atención e Hiperactividad (TDA/H). Tecnologías, actores sociales e industria farmacéutica. Physis, v. 1, n. 25, p. 75-98, 2015.

BIANCHI, E.; FARAONE, S. Diagnósticos y fármacos en la infancia: una perspectiva analítica desde las ciencias sociales. In: FARAONE, S.; BIANCHI, E. (comp.). Medicalización, salud mental e infancias: perspectivas y debates desde las ciencias sociales. Investigaciones acerca de Argentina y el sur de América Latina. Buenos Aires: Teseo, 2018. p. 61-92.¿

BIANCHI, E.; FARAONE, S.; ORTEGA, F; GONÇALVES, V. P.; ZORZANELLI, R. T. Controversias acerca del diagnóstico de TDAH y la prescripción de metilfenidato en los debates sobre la medicalización en Argentina y Brasil. Physis, v. 3, n. 27, p. 641-660, 2017.

BIANCHI, E.; ORTEGA, F.; FARAONE, S.; GONÇALVES, V. P.; ZORZANELLI, R. T. Medicalización más allá de los médicos. Marketing farmacéutico en torno al Trastorno por Déficit de Atención e Hiperactividad en Argentina y Brasil (1998-2014). Saúde \& Sociedade, n. 25, p. 452-62, 2016.

BIANCHI, E.; FARAONE, S.; OBERTI, M.; LEONE, C. Medicalización como problema de salud internacional. La prensa escrita online sobre TDAH en Argentina (2001-2017). Astrolabio, v. 1, n. 24, p. 17-51, 2020.

BUSFIELD, J. Pills, Power, People: Sociological Understandings of the Pharmaceutical Industry, Sociology, v. 2, n. 40, p. 297-314, 2006. 
CARBAJAL, M. ADD y ADHD, una mirada desde el periodismo. In: BENASAYAG, L. (comp.) ADDH. Nińos con déficit de atención e hiperactividad. ¿¿Una patología de mercado? Una mirada alternativa con enfoque multidisciplinario. Argentina: Noveduc, 2007. p. 237-244 i

CENTROS PARA EL CONTROL Y LA PREVENCIÓN DE ENFERMEDADES (CDC). Datos sobre el TDAH. 2018. Disponible en: http://www.cdc.gov/ncbddd/spanish/adhd/facts.html.

CEA D’ANCONA, M. Metodología Cuantitativa: estrategias y técnicas de investigación social. Madrid: Síntesis, 1996.

CLARKE, A. E.; SHIM, J. Medicalization and biomedicalization Revisited: technoscience and transformations of health, illness and American medicine. In: PESCOSOLIDO, B. A.; MARTIN, J. K.; MCLEOD, J. D.; ROGERS, A. (ed.). Handbook of the sociology of health, illness, and healing. A Blueprint for the 21st Century. New York: Springer; 2011. p. 173-195 ¿

CONRAD, P. The discovery of hyperkinesis. Notes on the medicalization of deviant behavior. Social Problems, v. 1, n. 23, p. 12-21, 1975.

CONRAD, P. Sobre la medicalización de la anormalidad y el control social. In: INGLEBY, D. (ed.). Psiquiatría Crítica. La política de la salud mental. Barcelona: Grijalbo, 1982. p. 129-154 ¿

CONRAD. P. The shifting engines of medicalization. Journal of Health and Social Behaviour, v. 1, n. 46, p. 3-14, 2005.

CONRAD, P. Medicalization: changing contours, characteristics, and contexts. In: COCKERHAM, W. (ed.). Medical sociology on the move: new directions in theory. New York: Springer, 2013.

CONRAD, P.; BERGEY, M. The impending globalization of ADHD: Notes on the expansion and growth of a medicalized disorder. Social Science \& Medicine, n. 122, p. 31-43, 2014.

CONRAD, P.; POTTER, D. From hyperactive children to ADHD adults. Observations on the expansion of medical categories. In: CONRAD, P.; LEITER, V. (ed.). Health and health care as social problems. New York: Rowman \& Littlefield, 2003. p. 39-65;

CONRAD, P.; SCHNEIDER, J. W. Deviance and Medicalization. From badness to sickness. Philadelphia: Temple University, 1992.

FARAONE, S. Reformas en salud mental. Dilemas en torno a las nociones, conceptos y tipificaciones, Salud Mental y Comunidad, n. 3, p. 29-40, 2013.

FARAONE, S; BIANCHI, E. The Journey of ADHD in Argentina: From the Increase in Methylphenidate Use to Tensions among Health Professionals. In: BERGEY, M.; FILIPE, A.; CONRAD, P.; SINGH, I. (ed.). Global Perspectives on ADHD. Social Dimensions of Diagnosis and Treatment in Sixteen Countries. Baltimore: Johns Hopkins University Press, 2018. p. 162-185 ¿

FARAONE, S.; ARIZAGA, C.; BARCALA, A.; TORRICELLI, F.; BIANCHI, E.; BALLESTEROS, I. Campo Salud. In: ARIZAGA, C.; FARAONE, S. La medicalización de la infancia. Nińos, escuela y psicotrópicos. Buenos Aires, SEDRONAR-II.GG. 2008. p. 163-237 ¿ 
Medicalización del TDAH en Argentina. Reflexiones sobre tendencias globales y especificidades locales a través del estudio del metilfenidato y la atomoxetina | Bianchi, Eugenia Faraone, Silvia A. Torricelli, Flavia C.

FARAONE, S.; BIANCHI, E. (comp.). Medicalización, salud mental e infancias: perspectivas y debates desde las ciencias sociales. Investigaciones acerca de Argentina y el sur de América Latina. Buenos Aires: Teseo, 2018.

FARAONE, S. V.; SERGEANT, J.; GILLBERG, C.; BIEDERMAN, J. The worldwide prevalence of ADHD: is it an American condition? Journal of World Psychiatry, v. 2, n. 2, p. 104-113, 2003.

GREENE, J.; SISMONDO, S. (ed.). The Pharmaceutical Studies Reader. New Jersey: Wiley Blackwell, 2015.

HEALY, D. The Creation of Psychopharmacology. London: Harvard University Press, 2002.

HERNÁNDEZ SAMPIERI, R.; FERNÁNDEZ COLLADO, C.; BAPTISTA LUCIO, P. Metodología de la investigación. Mc Graw Hill: México, 1998.

JENKINS, J. Psychopharmaceutical Self and Imaginary in the social field of Phychiatric Treatment. In: JENKINS, J. (ed.). Pharmaceutical Self. The Global Shaping of Experience in an Age of Phychopharmacology. Santa Fe: SAR, 2011. p. 17-40;

JUNTA INTERNACIONAL DE FISCALIZACIÓN DE ESTUPEFACIENTES (JIFE). Informe 1995. Disponible en: http:/www.incb.org/documents/Publications/AnnualReports/ AR1995/AR_1995_S.pdf Acceso en: 1 jul. 2020.

JUNTA INTERNACIONAL DE FISCALIZACIÓN DE ESTUPEFACIENTES (JIFE). Informe 2006. Available from: http:/www.incb.org/documents/Publications/AnnualReports/ AR2006/AR_2006_Spanish.pdf. Acceso en: 1 jul. 2020.

JUNTA INTERNACIONAL DE FISCALIZACIÓN DE ESTUPEFACIENTES (JIFE). Informe 2012. Disponible en: http://www.incb.org/documents/Publications/AnnualReports/ AR2012/AR_2012_S.pdf. Acceso en: 1 jul. 2020.

JUNTA INTERNACIONAL DE FISCALIZACIÓN DE ESTUPEFACIENTES (JIFE). Informe 2014. Disponible en: https:/www.incb.org/documents/Publications/AnnualReports/ AR2014/Spanish/AR_2014_ESP.pdf. Acceso en: 1 jul. 2020.

JUNTA INTERNACIONAL DE FISCALIZACIÓN DE ESTUPEFACIENTES (JIFE). Informe 2015. Disponible en: https:/www.incb.org/documents/Publications/AnnualReports/ AR2015/Spanish/AR_2015_S.pdf. Acceso en: 1 jul. 2020.

JUTEL, A. Sociology of diagnosis: a preliminary review, Sociology of Health \& Illness, v. 2, n. 31, p. 278-299, 2009.

JUTEL, A. Classification, disease, and diagnosis, Perspectives in Biology and Medicine, v. 2, n. 54, p. 189-205, 2011 a.

JUTEL, A. Putting a Name to it. Diagnosis in contemporary society. Baltimore: Johns Hopkins University Press, 2011b.

JUTEL, A. Beyond The Sociology of Diagnosis. Sociology Compass, v. 9, n. 9, p. 841-852, 2015.

MANIOWICZ, D. 2011. Malos diagnósticos y sobremedicación. La infancia en peligro. Revista Veintitrés, Buenos Aires, p. 27-29, 16 ju. 2011. 
MAYES, R.; BAGWELL, C.; ERKULWATER, J. ADHD and the Rise in Stimulant Use Among Children. Harvard Review of Psychiatry, v. 3, n. 16, p. 151-66, 2008.

MAYES, R.; RAFALOVICH, A. Suffer the restless children: the evolution of ADHD and paediatric stimulant use, 199-80. History of Psychiatry, v. 4, n. 18, p. 435-57, 2007.

MURGUÍA, A.; ORDORIKA, T.; GUERRERO, F. Consideraciones epistemológicas en torno a la medicalización en América Latina: balances y propuestas. Ludus Vitalis, XXIV, 46, p. 99-128, 2016.

MURGUÍA, A.; ORDORIKA, T.; LENDO, L. El estudio de los procesos de medicalización en América Latina. Manguinhos Historia Ciências Saúde, v. 3, n. 23, p. 635-51, 2016.

PETRYNA, A.; LAKOFF, A.; KLEINMAN, A. (ed.). Global pharmaceuticals. Ethics, markets, practices. Durkham and London: Duke, 2006.

POLANCYK, G.; DE LIMA, M. S.; HORTA, B. L.; BIEDERMAN, J.; RODHE, L. A. The worldwide prevalence of ADHD: a systematic review and metaregression analysis. American Journal of Psychiatry, v. 6, n. 164, p. 942-948. 2007.

REED, G.; CORREIA, J.; ESPARZA, P.; SAXENA, S.; MAJ, M. The WPA-WHO Global Survey of Psychiatrists' Attitudes Towards Mental Disorders Classification, World Psychiatry, n. 10, p. 118-131, 2011.

ROSE, N. Psychiatry as a political science: advanced liberalism and the administration of risk, History of Human Sciences, v. 2, n. 9, p. 1-23, 1996.

ROSE, N.; ABI-RASCHED, J. Neuro: The New Brain Sciences and the Management of the Mind. Princeton-Oxford: Princeton University Press, 2013.

SHORTER, E. A Historical Dictionary of Psychiatry. New York: Oxford University Press, 2005. SINGH, I. Beyond Polemics: Science and ethics of ADHD. Nature, v. 9, n. 12, p. 957-964, 2008. SINGH, I.; FILIPE, A. M.; BARD, I.; BERGEY, M.; BAKER, L. Globalization and cognitive enhancement: emerging social and ethical challenges for ADHD clinicians. Current Psychiatry Reports, v. 9, n. 15, p. 385, 2013.

WHITAKER, R. Anatomia de uma epidemia. Manguinhos: Fiocruz Editora, 2017.

WILLIAMS, S.; MARTIN, P.; GABE, J. The pharmaceuticalisation of society? A framework for analysis. Sociology of Health and Illnes, v. 5, n. 33, p. 710-725. 2011. 


\section{ADHD medicalization in Argentina. Reflections on global trends and local specificities through the study of methylphenidate and atomoxetine}

\section{Abstract}

The general objective is to contribute to a panoramic view of the phenomenon of the medicalization of ADHD in Argentina, and to discussions about the dissemination of knowledge about childhood. The analysis is part of studies on the transformation and expansion of medicalization in the XXI century, and the processes of diagnosis and treatment of ADHD are addressed. The article presents data from I) world production and consumption of methylphenidate, and data from Argentina from 2) import of methylphenidate as monodrug (2003-2016); 3) dispensed containers of atomoxetine and methylphenidate (2005-2010 and 2012-2016). These data are triangulated with qualitative sources: 84 semi-structured interviews, individual and group, between 2007 and 2017, to health professionals with clinical, teaching and research activities in the City of Buenos Aires, Tierra del Fuego, Corrientes and Salta. Consonant and dissonant Argentine trends with these processes are described and analyzed worldwide.

Keywords: Attention deficit hyperactivity disorder. Methylphenidate. Atomoxetine. Argentina.

\section{Medicalização do TDAH na Argentina. Reflexões sobre tendências globais e especificidades locais através do estudo do metilfenidato e atomoxetina}

\section{Resumo}

O objetivo geral é contribuir para uma visão panorâmica do fenômeno da medicalização do TDAH na Argentina e para discussões sobre a disseminação do conhecimento sobre a infância. $A$ análise faz parte de estudos sobre a transformação e expansão da medicalização no século XXI, e são abordados os processos de diagnóstico e tratamento do TDAH. O artigo apresenta dados de I) produção e consumo mundiais de metilfenidato e dados da Argentina de 2) importação de metilfenidato como monodrogas (2003-2016); 3) recipientes dispensados de atomoxetina e metilfenidato (2005-2010 e 2012-2016). Esses dados são triangulados com fontes qualitativas: 84 entrevistas semiestruturadas, individuais e em grupo, entre 2007 e 2017, a profissionais de saúde com atividades clínicas, de ensino e pesquisa na cidade de Buenos Aires, Terra do Fogo, Corrientes e Salta. Tendências argentinas consonantes e dissonantes com esses processos são descritas e analisadas em todo o mundo.

Palavras-chave: Transtorno de déficit de atenção e hiperatividade. Metilfenidato. Atomoxetina. Argentina. 